\title{
Boron pile-up phenomena during ultra shallow junction formation
}

\author{
M. Ferri ${ }^{(a)}$, S. Solmi ${ }^{(a)}$, D. Giubertoni ${ }^{(b)}$, M. Bersani ${ }^{(b)}$, J. J. Hamilton ${ }^{(c)}$, M. Kah ${ }^{(c)}$, N.E.B. Cowern ${ }^{(c)}$, K. \\ Kirkby $^{(\mathrm{c})}$, and E.J.H. Collart ${ }^{(\mathrm{d})}$
}

\begin{abstract}
(a) Institute of Microelectronics and Microsystems CNR-IMM Via Gobetti, 101 - 40129 Bologna, Italy.
${ }^{(b)}$ Fondazione Bruno Kessler - irst Via Sommarive, 18 - Povo, Trento 38050, Italy.

(c)Ion Beam Centre, Advance Tech. Institute, University of Surrey, Guildford, Surrey, GU2 7XH, UK.

${ }^{(d)}$ Parametric and Conductive Impl. Division, Applied Materials UK Ltd, Foundry Lane, Horsham, West

Sussex, RH13 5PX.
\end{abstract}

The redistribution during annealing of low-energy B implants in SOI structures and in bulk Si have been investigated by comparing Secondary Ion Mass Spectrometry (SIMS) and simulated profiles. Samples preamorphised with $\mathrm{Ge}$ at different implantation energies have been prepared in order to investigate the effects of the damage position on B diffusion. The specimens have been subsequently B implanted at 500 $\mathrm{eV}$ with doses $2 \times 10^{13}$ and $2 \times 10^{14} \mathrm{~cm}^{-2}$ and annealed between 700 and $1100{ }^{\circ} \mathrm{C}$. SIMS profiles show a B pile-up in the first few nanometres of the $\mathrm{Si}$ matrix on the $\mathrm{Si}$ surface. Simulations of diffused profiles indicate that the B redistribution upon annealing can be explained by assuming that the mobility of the dopant which arrives in proximity of the surface is practically annulled. The amount of B trapped at the surface is maximum at the temperatures around $800^{\circ} \mathrm{C}$, when more than $80 \%$ of the implanted dopant is made immobile and electrically inactive. The trapped $\mathrm{B}$ increases with reducing the depth of the amorphous layer and it is higher in the bulk Si than in SOI. By comparing Hall measurements and the amount of B not trapped at the surface, we also estimate the amount of B that aggregates inside the $\mathrm{Si}$ lattice in form of clusters (BICs). For the B dose of $2 \times 10^{14} \mathrm{~cm}^{-3}$, after isochronal annealing of $60 \mathrm{~s}$, the amount of BICs is about $3-4 \times 10^{13} \mathrm{~cm}^{-2}$ at the lowest temperatures and tends to vanish at high temperatures.

\section{Introduction}

Scaling of electronic devices requires both the reduction of all the physical dimensions, including junction depth profile, and high levels of active dopant concentration. Highly active, shallow and steep doping profile can be obtained by using preamorphising implants prior dopant implantation and annealing at low temperature exploiting the solid phase epitaxial re-growth
(SPER) process [1]. It is well known that, as a consequence of the amorphising implant, an excess of $\mathrm{Si}$ interstitials forms just beyond the amorphous/crystalline interface in the end-ofrange (EOR) region. During subsequent annealing these defects evolve through the formation/dissolution of aggregates and the release of free self interstitials [1-4]. This interstitial supersaturation causes unwanted phenomena like transient enhanced diffusion 
(TED), point defects clusters and clustering between point defects and dopant with consequent electrical deactivation [1,2].

The surface (and the interfaces) affect the impurity profile both through the segregation and trapping of dopant atoms in energetically favourite places and acting on point defects distribution, and consequently, on the TED phenomena. These effects are particularly evident for boron [5-8] and phosphorous [9, 10], but have been also evidenced in ultrashallow junctions obtained by low energy arsenic implantation [11, 12].

In these last years the interest for silicon-oninsulator (SOI) technology has strongly increased due to the expected superior performances and fabrication advantages of this material with respect to the bulk silicon [13]. As far as the diffusion phenomenon is concerned, the presence in the SOI samples of a buried $\mathrm{Si} / \mathrm{SiO}_{2}$ interface provides another efficient sink for the interstitials besides the silicon surface. This second sink modifies the distribution of the point defects during the annealing and consequently the unwanted effects related to these defects [14].

Purpose of this work is to investigate the uphill diffusion in ultrashallow $\mathrm{p}^{+} / \mathrm{n}$ junctions obtained by low energy B implantation in both bulk $\mathrm{Si}$ and $\mathrm{SOI}$ material. In particular, the dependence of this phenomenon on implanted dose, depth of the amorphous layer and annealing conditions has been analyzed with the support of a simulation program which takes into account the dopant segregation at the surface and allows a satisfactory describe of the dopant redistribution upon annealing.

\section{Experimental}

The experiments were performed on (100) oriented, n-type $\mathrm{CZ}$ silicon wafers with resistivity $10-25 \Omega . \mathrm{cm}$, and on SOI wafers with a $55 \mathrm{~nm}$-thick p-type $\mathrm{Si}$ over layer obtained by wafer bonding technology. The samples were $\mathrm{Ge}$ implanted to produce amorphous layers of different depth. With a Ge dose of $1 \times 10^{15} \mathrm{~cm}^{-2}$ and energies of 8,20 and $25 \mathrm{keV}$ we amorphised the silicon to depths of about, 20,40 and $45 \mathrm{~nm}$, respectively [15]. The specimens were subsequently B implanted at $500 \mathrm{eV}$ to doses of $2 \times 10^{13}$ and $2 \times 10^{14} \mathrm{~cm}^{-2}$. The annealing treatment were performed at temperatures between 700 and $1100{ }^{\circ} \mathrm{C}$ for different times between $5 \mathrm{~s}$ and $4 \mathrm{~h}$ using a Process Products Corporation 18 Lamp rapid thermal processing (RTP) annealer in nitrogen atmosphere. To monitor the temperature in the annealer, we use a k-type thermocouple attached to the bottom of a 5" Si support wafer and the samples to be annealed are placed on top of this support wafer. From the comparison of the re-growth rate of the amorphous layer we found that the bulk Si samples experienced abaut $3{ }^{\circ} \mathrm{C}$ higher temperature than the SOI specimens. This is within the uncertainties of the techniques used.

CAMECA Sc-Ultra mass spectrometer has been used to perform Secondary Ion Mass 
Sprectroscopy (SIMS) depth profiles. An oblique incidence $\left(68^{\circ}\right) 500 \mathrm{eV} \mathrm{O}{ }_{2}^{+}$primary beam has been used and positive secondary ions were collected. In order to obtain a suitable depth resolution and sensitivity a controlled oxygen leak was introduced in the analysis chamber and sample underwent a rotation during sputtering [16].

The electrical activity of the B implants was assessed by sheet resistance and Hall measurements using an Accent HL5500 machine.

The simulations of the diffused profiles are performed by using a pair-diffusion model which considers unpaired point defects and dopantdefect pairs as mobile species and the unpaired dopant on lattice sites as immobile species. To take into account the $\mathrm{B}$ uphill diffusion toward the surface we introduce a "trap" distribution into the $\mathrm{Si}$ in the first 2-3 nanometres below the $\mathrm{Si} /$ gas interface. All the simulations are performed with an equilibrium constant reaction which favours a rapid formation of the B-Trap complexes and with the trap density used as fitting parameter. This simple approach allows us to strongly improve the agreements between simulation and measured profiles in a wide range of experimental conditions. A more detailed description of the model can be found in Ref. [17]

\section{Results and discussion}

Fig. 1 shows the SIMS profiles of Ge PAI samples at $20 \mathrm{keV}$ and $\mathrm{B}$ implanted with dose $2 \times 10^{13} \mathrm{~cm}^{-2}$ after annealing for $60 \mathrm{~s}$ at various temperatures both in SOI (Fig. 1a) and in bulk material (Fig. 1b). In dotted line the simulated profiles are also reported. SIMS profiles clearly show a B pile-up in the first few nanometres of the Si matrix on the Si surface. The simulations permit to describe the phenomenon of the uphill diffusion and to evaluate the amount of $B$ collected on the surface region.

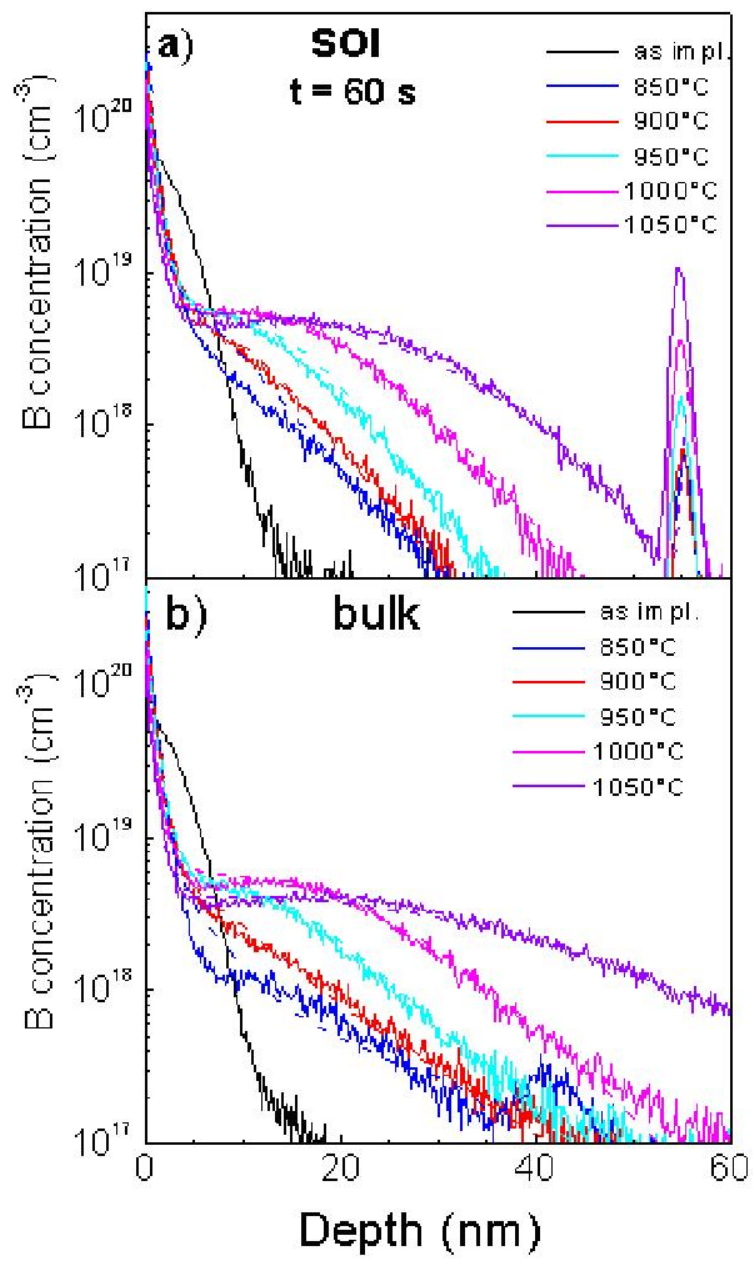

Fig. 1: Comparison between simulated (dashed lines) and SIMS (continuous lines) profiles of PAI samples at $20 \mathrm{keV}$, B implanted with dose $2 \times 10^{13} \mathrm{~cm}^{-2}$ after isochronal annealing of $60 \mathrm{~s}$ at various temperatures in SOI (Fig. 1a) and in bulk material (Fig. 1b). 
Simulations indicate that the B diffusion, apparently against the concentration gradient, is consistent with Fick's diffusion equation if the diffusivity near the surface suddenly drops to negligible values; this effect is performed in the simulation by assuming the presence of traps in that region without considering any asymmetric behaviour of the dopant movement. The presence of unsaturated traps in the surface region drives the $[\mathrm{BI}]$ pair concentration to be very small, and thus creates a huge gradient between the $\mathrm{B}$ profile and the traps region. This gradient drives the phenomenon of the uphill diffusion. On the contrary, simulations performed without the introduction of traps near the surface bring to a relevant overestimation of the junction depth and an incorrect determination of the $\mathrm{B}$ concentration in the surface region (not shown).

By using the SIMS profiles and simulations the amount of $\mathrm{B}$ which is distributed into the $\mathrm{Si}$, and not trapped at the surface, can be evaluated. The obtained results are reported in Fig. 2 for the $\mathrm{B}$ implanted dose of $2 \times 10^{13} \mathrm{~cm}^{-2}$ as a function of the annealing temperature for a $60 \mathrm{~s}$ treatment. The data refer to the bulk samples preamorphised with $\mathrm{Ge}$ implantation at 8 and $20 \mathrm{KeV}$. In all samples there is a minimum in the free-B amount around $800-850{ }^{\circ} \mathrm{C}$; at these temperatures more than $80 \%$ of the implanted B is trapped at the surface.

Boron segregation at the surface depends on annealing temperature. At low temperatures (700$775^{\circ} \mathrm{C}$ ) the low $\mathrm{B}$ diffusivity limits the dopant atoms to reach the surface then the segregation is reduced. This is confirmed by the dependence of the trapped B amount on the position of the EOR defects. Higher trapping is obtained in the samples where the dissolving EOR defects, which release interstitials and promote $\mathrm{B}$ diffusion, are nearer to the surface. At high annealing temperature, B segregated at the surface reduces.

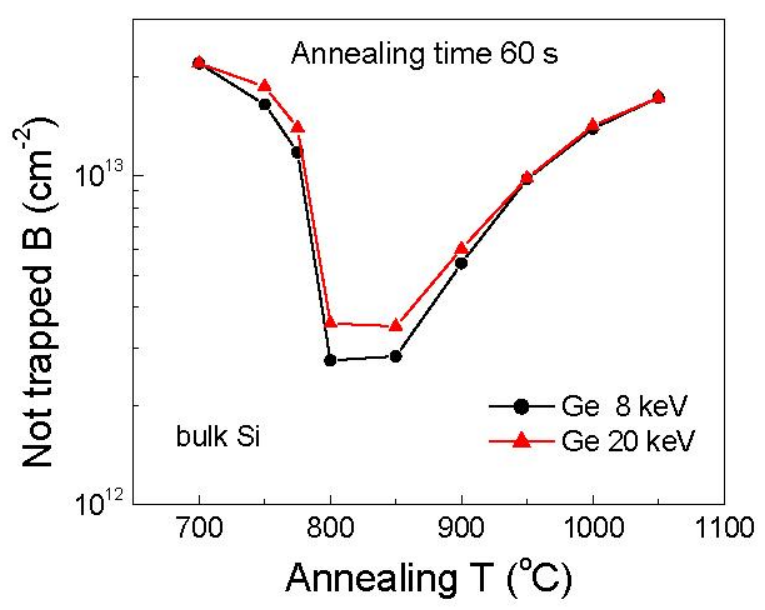

Fig. 2: Amount of not trapped $B$ vs annealing temperature for isochronal treatments of $60 \mathrm{~s}$ in bulk samples PAI with Ge at different energies, in order to locate the EOR defects at different depths. The B implanted dose was $2 \times 10^{13} \mathrm{~cm}^{-2}$.

This is due both to the lowering of interstitial supersaturation with increasing temperature that allows for $B$ atoms to avoid the surface segregation diffusing inside the bulk and to the tendency of segregation phenomena to reduce at higher temperature. However, only for temperature as higher than $1050^{\circ} \mathrm{C}$ we have a nearly complete release of the trapped B (see Fig. 2).

The different evolution of the interstitial supersaturation is also responsible for the differences of B trapping between SOI and bulk 
Si shown in Fig. 3a and Fig. 4 for samples B implanted with dose $2 \times 10^{14} \mathrm{~cm}^{-2}$.

In fact, in SOI samples the buried oxide acts as a sink for interstitials increasing EOR dissolution rate and reducing the enhancement of the $B$ diffusion [7]. The effect is more evident for the samples preamorphised at $20 \mathrm{keV}$ due to the proximity of the EOR defects to the buried oxide layer. Also for this dose the surface trapping in the samples preamorphised at $8 \mathrm{keV}$ is higher respect to the one exhibited by the samples more deeply preamorphised at $20 \mathrm{keV}$.

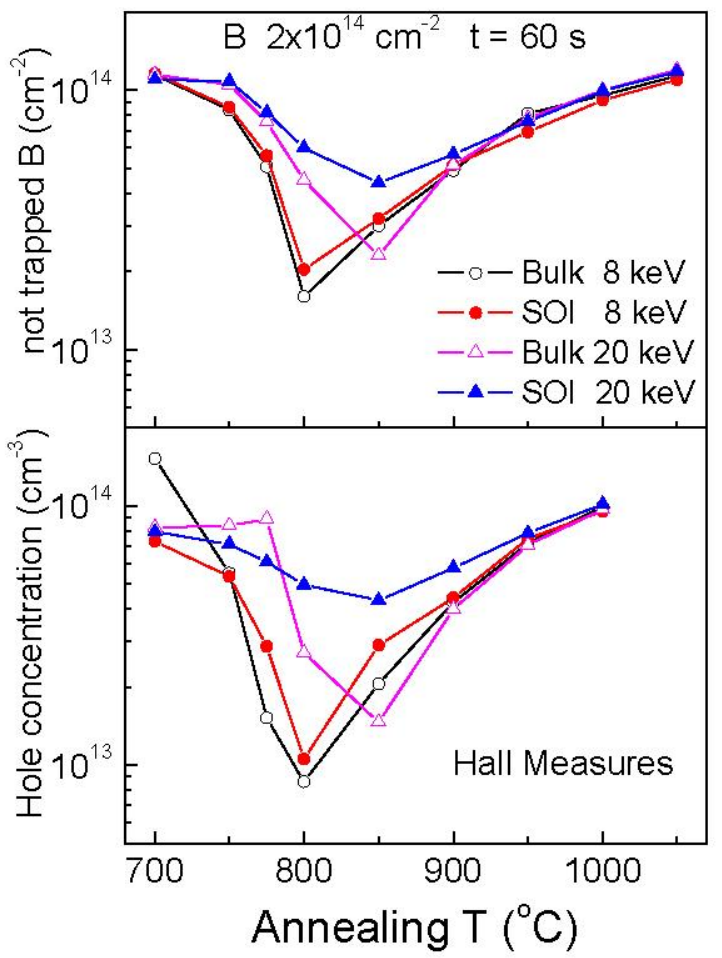

Fig. 3: Amount of not trapped B (3a) and hole concentration (3b) vs annealing temperature for isochronal treatments of $60 \mathrm{~s}$ in SOI samples and bulk material PAI with Ge at different energies. The $B$ implanted dose was $2 \times 10^{14} \mathrm{~cm}^{-2}$.

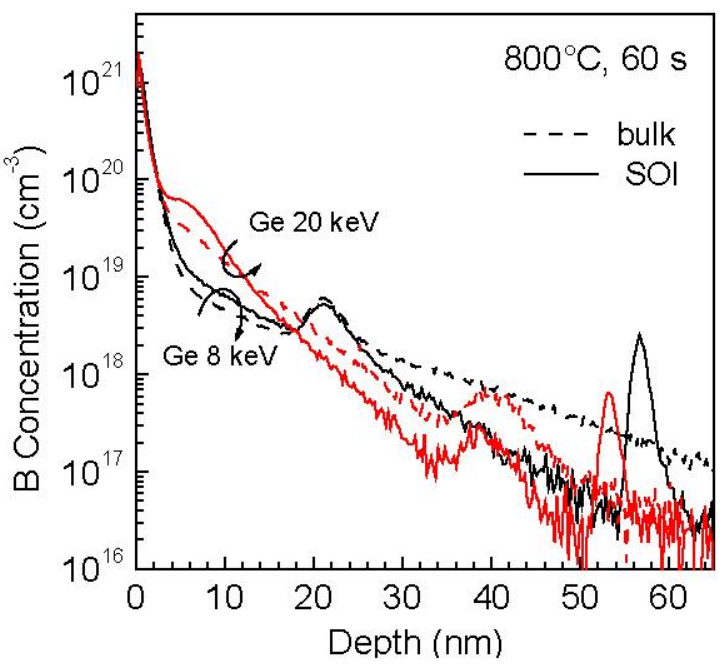

Fig. 4: Comparison of the $B$ profiles of samples implanted with dose $2 \times 10^{14} \mathrm{~cm}^{-2}$ and annealed at 800 ${ }^{\circ} \mathrm{C}$ for $60 \mathrm{~s}$ in SOI and bulk Si PAI with Ge at different energies.

The trend of the not trapped B vs temperature shown in Fig. 3a is in qualitative agreement with the one of the active $\mathrm{B}$ concentration reported in Fig 3b. Two effects contribute to the $B$ deactivation: i) $\mathrm{B}$ segregation in proximity of the surface and ii) formation of Boron interstitial clusters (BICs) inside the Si lattice in presence of high interstitial supersaturation [18]; both these contributions increase with increasing interstitial supersaturation. However, the good correlation between hole concentration and not trapped $\mathrm{B}$ indicates that surface segregation is the dominant effect to determine the amount of active $B$ in these experimental conditions.

Fig. 5 shows the comparison between the hole concentration and the not trapped boron of $\mathrm{Ge}$ PAI samples at $8 \mathrm{keV}$ and $\mathrm{B}$ implanted with dose $2 \times 10^{14} \mathrm{~cm}^{-2}$ after annealing for $60 \mathrm{~s}$ at various temperatures in the SOI material. The difference between the two curves, i.e. the amount of BICs 
present after $1 \mathrm{~min}$ annealing, is in the range 3$4 \times 10^{13} \mathrm{~cm}^{-2}$ at low temperatures and then reduces with increasing temperature down to vanish, in agreement with the dissolution (or not formation) of these clusters (see Fig.5). A value of $3-4 \times 10^{13}$ $\mathrm{cm}^{-2}$ means that at low temperature about $30-40 \%$ of the B located inside the silicon is in form of BICs.

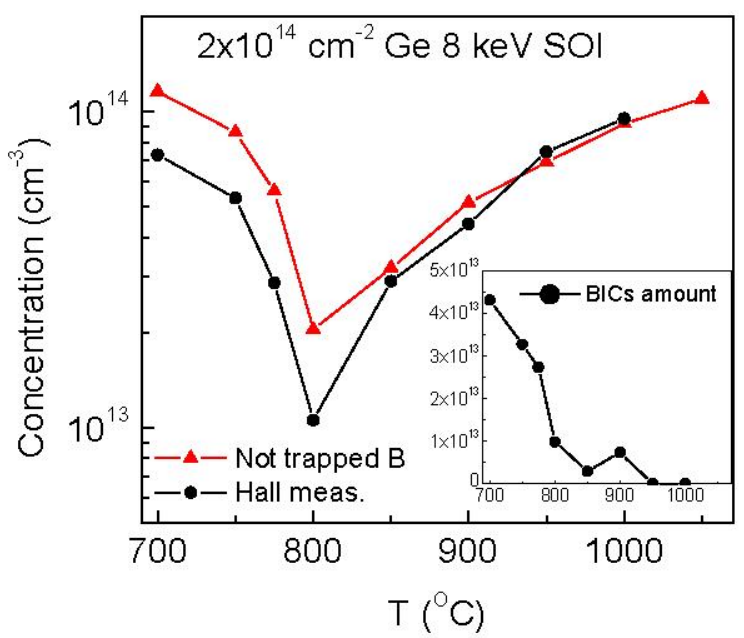

Fig. 5: Comparison between not trapped $B$ and hole concentration vs annealing temperature for isochronal treatment of $60 \mathrm{~s}$ in SOI sample PAI with Ge at $8 \mathrm{keV}$ and $B$ implanted at $2 \times 10^{14} \mathrm{~cm}^{-2}$.

The junction depths, $X_{j}$, evaluated from SIMS profiles at the concentration of $1 \times 10^{18} \mathrm{~cm}^{-3}$, for the samples B implanted with dose $2 \times 10^{14} \mathrm{~cm}^{-2}$ are reported in Fig. 6 as a function of the annealing temperature. For temperatures higher than $850^{\circ} \mathrm{C}$ a shallower $\mathrm{X}_{\mathrm{j}}$ in SOI material with respect to bulk $\mathrm{Si}$ is obtained in agreement with previous observations [19]. This effect was attributed to the interstitial recombination on the buried oxide layer [20, 19].

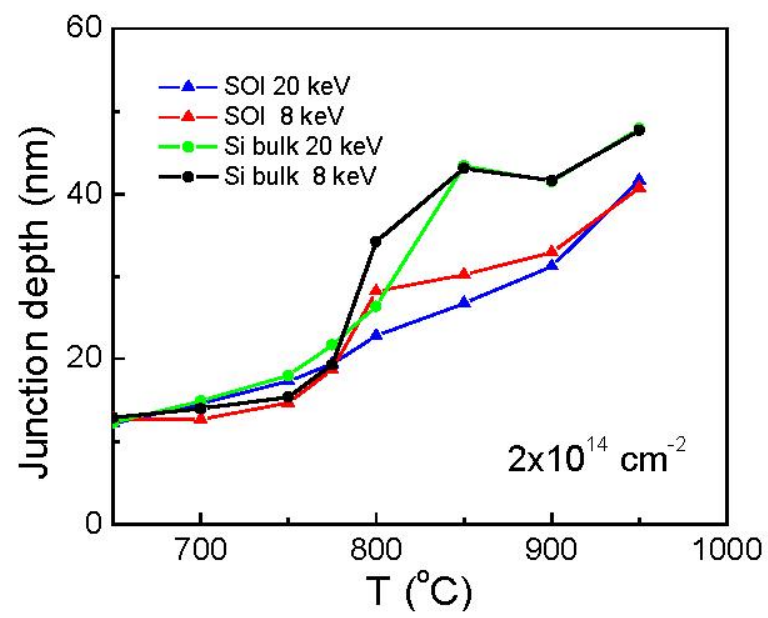

Fig. 6: Junction depths $\left(X_{j}\right)$, evaluated from SIMS profiles at the concentration of $1 \times 10^{18} \mathrm{~cm}^{-3}$, for the samples $B$ implanted with dose $2 \times 10^{14} \mathrm{~cm}^{-2}$ as a function of the annealing temperature for isothermal treatments of $60 \mathrm{~s}$. The figure shows the results of SOI and bulk Si PAI with Ge at 8 and $20 \mathrm{keV}$.

\section{Conclusions}

Ultrashallow junctions obtained by low energy $(500 \mathrm{eV}) \mathrm{B}$ implanted in preamorphised SOI and in bulk Si show, after annealing, a strong effect of uphill diffusion which confines, and makes electrically inactive, a relevant amount of dopant in proximity of the surface. This redistribution can be accounted for by simulations based on pair diffusion model and with the introduction of trapping sites in proximity of the surface, without considering any asymmetric behaviour of the dopant movement. The amount of trapped B increases with decreasing temperature, and, for a fixed temperature, is higher in the conditions of higher diffusivity. The correlation between trapped $\mathrm{B}$ and diffusivity allows explaining the 
increase of the trapped B with reducing the depth of the amorphous layer and the differences observed between SOI layers and bulk Si. At the temperatures around $800{ }^{\circ} \mathrm{C}$, more than $80 \%$ of the implanted dopant is trapped in the surface region.

By comparing Hall measurements and the amount of B not trapped at the surface, we estimate the amount of BICs formed during the annealing. For the B dose of $2 \times 10^{14} \mathrm{~cm}^{-3}$, after isochronal annealing of $60 \mathrm{~s}$, the amount of BICs is about $3-4 \times 10^{13} \mathrm{~cm}^{-2}$ at the lowest temperatures and tends to vanish at high temperatures.

\section{Acknowledgments}

This work was partially supported by the FIRB project (contract RBNE012N3X).

\section{Bibliography}

1. P. A. Stolk, J. H.-J. Gossmann, D. J. Eaglesham, D. C. Jacobson, C. S. Rafferty, G. H. Gilmer, M. Jariz, and J. M. Poate, J. Appl. Phys. 81, 6031 (1997).

2. M. Servidori, Z. Sourek, and S. Solmi, J. Appl. Phys. 62, 1723 (1987).

3. A. Clavere, B. Colombeau, B. De Mauduit, C. Bonafos, X. Herras, G. Ben Assayag, and F. Cristiano, Appl. Phys. A, 76, 1025 (2003).

4. C. Bonafos, D. Matoit, and A. Claveries, J. Appl. Phys. 83, 3008 (1998).

5. H. C. Wang, C. C. Wang, C. S. Chang, T. Wang, P. B. G, and C. H. Diaz, IEEE El. Dev. Lett. 29, 65 (2001).
6. A. Shima, T. Jinbo, N. Natsuaki, J. Ushio, J.-H. Oh, K. Ono, and M. Oshima, J. Appl. Phys. 89, 3458 (2001).

7. R. Duffy, V. C. Venezia, A. Heringa, T. W. T. Husken, M. J. P. Hopstaken, N. E. B. Cowern, P. B. Griffin, C. C. Wang, Appl. Phys. Lett. 82, 3647 (2003)

8. M. Y. L. Jung, R. Gunawan, R. D. Braatz, and E. G. Seebauer, J. Appl. Phys. 95, 1134 (2004).

9. R. Duffy, V. C. Venezia, J. Loo, M. J. P. Hopstaken, M. A. Verheijen, J. G. M. van Berkum, G. C. J. Maas, and C. Demeurisse, Appl. Phys. Lett. 86, 81917 (2005).

10. S. Ruffell, I. V. Mitchell, and P. J. Simpson, J. Appl. Phys. 97, 123518 (2005).

11. S. Solmi, M. Ferri, M. Bersani, D. Giubertoni, and V. Soncini, J. Appl. Phys. 94, 4950 (2003).

12. M. Ferri, S. Solmi, A. Parisini, M. Bersani, D. Giubertoni and M. Barozzi, J. App. Phys. 99, 113508 (2006).

13. G. K. Celler and S. Cristoloveanu, J. Appl. Phys. 93, 4955 (2003).

14. P. Fahey and S. Solmi, J. Appl. Phys. 60, 4329 (1986).

15. J. J. Hamilton, E. J. H. Collart, B. Colombeau, C. Jeynes, M. Bersani, D. Giubertoni, J. A. Sharp, N. E. B. Cowern, and K. J. Kirkby, Nuclear Instr. and Meth. B, 237, 107 (2005).

16. M. Bersani, D. Giubertoni, E. Iacob, M. Barozzi, S. Pederzoli, L. Vanzetti, M. Anderle, Appl. Surf. Sci. 252, 7315 (2006).

17. M. Ferri, S. Solmi, A. Parisini, M. Bersani, D. Giubertoni, and M. Barozzi, J. Appl. Phys. 99, 113508 (2006). 
18. B. J. Pawlak, R. Surdeanu, B. Colombeau, A. J. Smith, N. E. B. Cowern, R. Lindsay, W. Vandervorst, B. Brijs, O. Richard, and F. Cristiano, Appl. Phys. Lett. 84, 2055 (2004).

19. J. J. Hamilton, N. E. B. Cowern, J. A.Sharp, K. J. Kirkby, E. J. H. Collart, B. Colombeau, M. Bersani, D. Giubertoni, A. Parisini, Appl. Phys. Lett. 89, 042111(2006).
20. J. J. Hamilton, E. J. H. Collart, B. Colombeau, M. Bersani, D. Giubertoni, J. A. Sharp, N. E. B. Cowern, K. J. Kirkby, Mat. Science and Eng B., 124, 215 (2005). 